International Journal of Pure and Applied Mathematics

Volume 108 No. 1 2016, 141-158

ISSN: 1311-8080 (printed version); ISSN: 1314-3395 (on-line version)

url: http://www.ijpam.eu

doi: $10.12732 /$ ijpam.v108i1.13

\title{
SOME COMMON FIXED POINT RESULTS FOR COMPATIBLE MAPPINGS OF TYPES IN MULTIPLICATIVE METRIC SPACES
}

\author{
Young Chel Kwun ${ }^{1}$, Parveen Kumar ${ }^{2}$, Shin Min Kang ${ }^{3}$ §, Sanjay Kumar ${ }^{4}$ \\ ${ }^{1}$ Department of Mathematics \\ Dong-A University \\ Busan, 49315, KOREA \\ ${ }^{2,4}$ Departement of Mathematics \\ Deenbandhu Chhotu Ram University of Science and Technology \\ Murthal, Sonepat, 131039, Haryana, INDIA \\ ${ }^{3}$ Department of Mathematics and RINS \\ Gyeongsang National University \\ Jinju, 52828, KOREA
}

Abstract: In this paper, we proved the some common fixed point results for compatible mappings of types in multiplicative metric spaces.

AMS Subject Classification: $47 \mathrm{H} 10,54 \mathrm{H} 25$

Key Words: multiplicative metric spaces, compatible mappings, compatible mappings of type $(A)$, of type $(B)$, of type $(C)$, of type $(P)$

\section{Introduction and Preliminaries}

It is well know that the set of positive real numbers $\mathbb{R}_{+}$is not complete according to the usual metric. To overcome this problem, in 2008, Bashirov et al. [1] introduced the concept of multiplicative metric spaces as follows:

Definition 1.1. Let $X$ be a nonempty set. A multiplicative metric is a

Received: March 25, 2016

Published: June 6, 2016

${ }^{\S}$ Correspondence author
(C) 2016 Academic Publications, Ltd.

url: www.acadpubl.eu 
mapping $d: X \times X \rightarrow \mathbb{R}_{+}$satisfying the following conditions:

(i) $d(x, y) \geq 1$ for all $x, y \in X$ and $d(x, y)=1$ if and only if $x=y$;

(ii) $d(x, y)=d(y, x)$ for all $x, y \in X$;

(iii) $d(x, y) \leq d(x, z) \cdot d(z, y)$ for all $x, y, z \in X$ (multiplicative triangle inequality).

Then the mapping $d$ together with $X$, that is, $(X, d)$ is a multiplicative metric space.

Example 1.2. ([2]) Let $\mathbb{R}_{+}^{n}$ be the collection of all $n$-tuples of positive real numbers. Let $d^{*}: \mathbb{R}_{+}^{n} \times \mathbb{R}_{+}^{n} \rightarrow \mathbb{R}$ be defined as follows:

$$
d^{*}(x, y)=\left|\frac{x_{1}}{y_{1}}\right|^{*} \cdot\left|\frac{x_{2}}{y_{2}}\right|^{*} \cdots\left|\frac{x_{n}}{y_{n}}\right|^{*},
$$

where $x=\left(x_{1}, \ldots, x_{n}\right), y=\left(y_{1}, \ldots, y_{n}\right) \in \mathbb{R}_{+}^{n}$ and $|\cdot|^{*}: \mathbb{R}_{+} \rightarrow \mathbb{R}_{+}$is defined by

$$
|a|^{*}= \begin{cases}a & \text { if } a \geq 1, \\ \frac{1}{a} & \text { if } a<1 .\end{cases}
$$

Then it is obvious that all conditions of a multiplicative metric are satisfied. Therefore $\left(\mathbb{R}_{+}^{n}, d^{*}\right)$ is a multiplicative metric space.

One can refer to [2] for detailed a multiplicative metric topology.

Definition 1.3. Let $(X, d)$ be a multiplicative metric space. Then a sequence $\left\{x_{n}\right\}$ in $X$ said to be

(1) a multiplicative convergent to $x$ if for every multiplicative open ball $B_{\epsilon}(x)=\{y \mid d(x, y)<\epsilon\}, \epsilon>1$, there exists $N \in \mathbb{N}$ such that $x_{n} \in B_{\epsilon}(x)$ for all $n \geq N$, that is, $d\left(x_{n}, x\right) \rightarrow 1$ as $n \rightarrow \infty$.

(2) a multiplicative Cauchy sequence if for all $\epsilon>1$, there exists $N \in \mathbb{N}$ such that $d\left(x_{n}, x_{m}\right)<\epsilon$ for all $m, n \geq N$, that is, $d\left(x_{n}, x_{m}\right) \rightarrow 1$ as $n, m \rightarrow \infty$.

(3) We call a multiplicative metric space complete if every multiplicative Cauchy sequence in it is multiplicative convergent to $x \in X$.

In 2012, Özavsar and Çevikel [2] gave the concept of multiplicative contractive mappings and proved some fixed point theorem of such mappings in a multiplicative metric space. 
Definition 1.4. Let $f$ be a mapping of a multiplicative metric space $(X, d)$ into itself. Then $f$ is said to be a multiplicative contraction if there exists a real number $\lambda \in[0,1)$ such that

$$
d(f x, f y) \leq d^{\lambda}(x, y) \text { for all } x, y \in X .
$$

In 2015, Kang et al. [3] introduced the notion of compatible mappings and its variants as follows:

Definition 1.5. Let $f$ and $g$ be mappings of a multiplicative metric space $(X, d)$ Then $f$ and $g$ are called

(1) compatible if

$$
\lim _{n \rightarrow \infty} d\left(f g x_{n}, g f x_{n}\right)=1,
$$

whenever $\left\{x_{n}\right\}$ is a sequence in $X$ such that $\lim _{n \rightarrow \infty} f x_{n}=\lim _{n \rightarrow \infty} g x_{n}=t$ for some $t \in X$,

(2) compatible of type $(A)$ if

$$
\lim _{n \rightarrow \infty} d\left(f g x_{n}, g^{2} x_{n}\right)=1 \text { and } \lim _{n \rightarrow \infty} d\left(g f x_{n}, f^{2} x_{n}\right)=1
$$

whenever $\left\{x_{n}\right\}$ is a sequence in $X$ such that $\lim _{n \rightarrow \infty} f x_{n}=\lim _{n \rightarrow \infty} g x_{n}=t$ for some $t \in X$,

(3) compatible of type $(B)$ if

$$
\lim _{n \rightarrow \infty} d\left(f g x_{n}, g^{2} x_{n}\right) \leq\left[\lim _{n \rightarrow \infty} d\left(f g x_{n}, f t\right) \cdot \lim _{n \rightarrow \infty} d\left(f t, f^{2} x_{n}\right)\right]^{1 / 2}
$$

and

$$
\lim _{n \rightarrow \infty} d\left(g f x_{n}, f^{2} x_{n}\right) \leq\left[\lim _{n \rightarrow \infty} d\left(g f x_{n}, g t\right) \cdot \lim _{n \rightarrow \infty} d\left(g t, g^{2} x_{n}\right)\right]^{1 / 2},
$$

whenever $\left\{x_{n}\right\}$ is a sequence in $X$ such that $\lim _{n \rightarrow \infty} f x_{n}=\lim _{n \rightarrow \infty} g x_{n}=t$ for some $t \in X$,

(4) compatible of type $(C)$ if

$\lim _{n \rightarrow \infty} d\left(f g x_{n}, g^{2} x_{n}\right) \leq\left[\lim _{n \rightarrow \infty} d\left(f g x_{n}, f t\right) \cdot \lim _{n \rightarrow \infty} d\left(f t, f^{2} x_{n}\right) \cdot \lim _{n \rightarrow \infty} d\left(f t, g^{2} x_{n}\right)\right]^{1 / 3}$

and

$\lim _{n \rightarrow \infty} d\left(g f x_{n}, f^{2} x_{n}\right) \leq\left[\lim _{n \rightarrow \infty} d\left(g f x_{n}, g t\right) \cdot \lim _{n \rightarrow \infty} d\left(g t, g^{2} x_{n}\right) \cdot \lim _{n \rightarrow \infty} d\left(g t, f^{2} x_{n}\right)\right]^{1 / 3}$,

whenever $\left\{x_{n}\right\}$ is a sequence in $X$ such that $\lim _{n \rightarrow \infty} f x_{n}=\lim _{n \rightarrow \infty} g x_{n}=t$ for some $t \in X$, 
(5) compatible of type $(P)$ if

$$
\lim _{n \rightarrow \infty} d\left(f^{2} x_{n}, g^{2} x_{n}\right)=1
$$

whenever $\left\{x_{n}\right\}$ is a sequence in $X$ such that $\lim _{n \rightarrow \infty} f x_{n}=\lim _{n \rightarrow \infty} g x_{n}=t$ for some $t \in X$.

Now we give some properties related to compatible mappings and its variants in a multiplicative metric space, see [3].

Proposition 1.6. Let $f$ and $g$ be compatible mappings of type $(A)$ of a multiplicative metric space $(X, d)$ into itself. If one of $f$ and $g$ is continuous, then $f$ and $g$ are compatible.

Proposition 1.7. Let $f$ and $g$ compatible mappings of type $(B)$ of a multiplicative metric space $(X, d)$ into itself. If $f t=g t$ for some $t \in X$, then $f g t=f f t=g g t=g f t$.

Proposition 1.8. Let $f$ and $g$ compatible mappings of type $(B)$ of a multiplicative metric space $(X, d)$ itself. Suppose that $\lim _{n \rightarrow \infty} f x_{n}=\lim _{n \rightarrow \infty} g x_{n}=$ $t$ for some $t \in X$. Then:

(i) $\lim _{n \rightarrow \infty} g g x_{n}=f t$ if $f$ is continuous at $t$.

(ii) $\lim _{n \rightarrow \infty} f f x_{n}=g t$ if $g$ is continuous at $t$.

(iii) $f g t=g f t$ and $f t=g t$ if $f$ and $g$ are continuous at $t$.

Remark 1.9. (1) In Proposition 1.7, assume that $f$ and $g$ are compatible mappings of type $(C)$ or of type $(P)$ instead of type $(B)$. Then the conclusion of Proposition 1.7 still holds.

(2) In Proposition 1.8, assume that $f$ and $g$ are compatible mappings of type $(C)$ or of type $(P)$ instead of type $(B)$. Then the conclusion of Proposition 1.8 still holds.

\section{Main Results}

Now we give the following theorem for compatible mappings of type $(A)$. 
Theorem 2.1. Let $A, B, S$ and $T$ be mappings of a complete multiplicative metric space $(X, d)$ satisfying the following conditions

$$
S X \subset B X, \quad T X \subset A X
$$

$$
d(S x, T y) \leq M^{\lambda}(x, y)
$$

for each $x, y \in X$ and $\lambda \in(0,1 / 2)$, where

$$
\begin{aligned}
& M(x, y) \\
& =\max \left\{d(A x, S x), d(B y, T y), d(B y, A x),(d(A x, T y) \cdot d(B y, S x))^{1 / 2},\right. \\
& \quad \min \left\{\frac{d(A x, S x) \cdot d(B y, T y)}{d(A x, B y)}, \frac{d(A x, T y) \cdot d(B y, S x)}{d(A x, B y)},\right. \\
& \left.\left.\quad \frac{d(A x, T y) \cdot d(B y, S x)}{d(S x, T y)}\right\}\right\} ;
\end{aligned}
$$

$\left(C_{3}\right)$ one of $A, B, S$ and $T$ is continuous;

Assume that the pairs $A, S$ and $B, T$ are compatible of type $(A)$. Then $A, B, S$ and $T$ have a unique common fixed point in $X$.

Proof. Suppose that $S$ is continuous. Since $A$ and $S$ are compatible of type $(A)$, by Proposition 1.6, $A$ and $S$ are compatible. The result easily follows from [4, Theorem 2.1].

Similarly, we can complete the proof when $A$ or $B$ or $T$ is continuous.

Next we give the following theorem for compatible mappings of type $(B)$.

Theorem 2.2. Let $A, B, S$ and $T$ be mappings of a complete multiplicative metric space $(X, d)$ into itself satisfying the conditions $\left(C_{1}\right),\left(C_{2}\right)$ and $\left(C_{3}\right)$.

Assume that the pairs $A, S$ and $B, T$ are compatible of type $(B)$. Then $A, B, S$ and $T$ have a unique common fixed point in $X$.

Proof. Let $x_{0} \in X$ be an arbitrary point. Since $S X \subset B X$ and $T X \subset A X$, there exists $x_{1} \in X$ such that $S x_{0}=B x_{1}=y_{0}$ and for this point $x_{1}$, there exists $x_{2} \in X$ such that $T x_{1}=A x_{2}=y_{1}$. Continuing in this way, we can construct a sequence $\left\{y_{n}\right\}$ such that

$$
y_{2 n+1}=T x_{2 n+1}=A x_{2 n+2} ; \quad y_{2 n}=S x_{2 n}=B x_{2 n+1} .
$$


From the proof of [4, Theorem 2.1], $\left\{y_{n}\right\}$ is a multiplicative Cauchy sequence in $X$ and hence it converges to some point $z \in X$. Further the subsequence $\left\{S x_{2 n}\right\},\left\{A x_{2 n}\right\},\left\{T x_{2 n+1}\right\}$ and $\left\{B x_{2 n+1}\right\}$ of $\left\{y_{n}\right\}$ converges to $z$.

Suppose that $S$ is continuous. Then we have

$$
S S x_{n} \rightarrow S z, \quad S A x_{2 n} \rightarrow S z \quad \text { as } n \rightarrow \infty
$$

Since $A$ and $S$ are compatible of type $(B)$, by Proposition 1.8 , Hence we have

$$
A A x_{2 n} \rightarrow S z \quad \text { as } n \rightarrow \infty .
$$

Now putting $x=A x_{2 n}$ and $y=x_{2 n+1}$ in $\left(C_{2}\right)$, we have

$$
d\left(S A x_{2 n}, T x_{2 n+1}\right) \leq M^{\lambda}\left(A x_{2 n}, x_{2 n+1}\right)
$$

where

$$
\begin{aligned}
& M\left(A x_{2 n}, x_{2 n+1}\right) \\
& =\max \left\{d\left(A A x_{2 n}, S A x_{2 n}\right), d\left(B x_{2 n+1}, T x_{2 n+1}\right), d\left(B x_{2 n+1}, A A x_{2 n}\right),\right. \\
& \left(d\left(A A x_{2 n}, T x_{2 n+1}\right) \cdot d\left(B x_{2 n+1}, S A x_{2 n}\right)\right)^{1 / 2}, \\
& \min \left\{\frac{d\left(A A x_{2 n}, S A x_{2 n}\right) \cdot d\left(B x_{2 n+1}, T x_{2 n+1}\right)}{d\left(A A x_{2 n}, B x_{2 n+1}\right)},\right. \\
& \quad \frac{d\left(A A x_{2 n}, T x_{2 n+1}\right) \cdot d\left(B x_{2 n+1}, S A x_{2 n}\right)}{d\left(A A x_{2 n}, B x_{2 n+1}\right)}, \\
& \left.\left.\quad \frac{d\left(A A x_{2 n}, T x_{2 n+1}\right) \cdot d\left(B x_{2 n+1}, S A x_{2 n}\right)}{d\left(S A x_{2 n}, T x_{2 n+1}\right)}\right\}\right\} .
\end{aligned}
$$

Taking $n \rightarrow \infty$, we get

$$
\begin{aligned}
& \lim _{n \rightarrow \infty} M\left(A x_{2 n}, x_{2 n+1}\right) \\
& =\max \left\{1,1, d(z, S z),(d(S z, z) \cdot d(z, S z))^{1 / 2},\right. \\
& \quad \min \{1 / d(S z, z), d(z, S z), d(z, S z)\}\} \\
& =d(S z, z) .
\end{aligned}
$$

Hence we have

$$
d(S z, z) \leq d^{\lambda}(S z, z)
$$

which implies that $S z=z$. Since $S X \subset B X$ there exists a point $u \in X$ such that $z=S z=B u$. 
Consider $x=A x_{2 n}$ and $y=u$ in $\left(C_{2}\right)$, we have

$$
d\left(S A x_{2 n}, T u\right) \leq M^{\lambda}\left(A x_{2 n}, u\right)
$$

where

$$
\begin{aligned}
& M\left(A x_{2 n}, u\right) \\
& =\max \left\{d\left(A A x_{2 n}, S A x_{2 n}\right), d(B u, T u), d\left(B u, A A x_{2 n}\right),\right. \\
& \left(d\left(A A x_{2 n}, T u\right) \cdot d\left(B u, S A x_{2 n}\right)\right)^{1 / 2}, \min \left\{\frac{d\left(A A x_{2 n}, S A x_{2 n}\right) \cdot d(B u, T u)}{d\left(A A x_{2 n}, B u\right)},\right. \\
& \left.\left.\quad \frac{d\left(A A x_{2 n}, T u\right) \cdot d\left(B u, S A x_{2 n}\right)}{d\left(A A x_{2 n}, B u\right)}, \frac{d\left(A A x_{2 n}, T u\right) \cdot d\left(B u, S A x_{2 n}\right)}{d\left(S A x_{2 n}, T u\right)}\right\}\right\} .
\end{aligned}
$$

Taking $n \rightarrow \infty$, we get

$$
\begin{aligned}
& \lim _{n \rightarrow \infty} M\left(A x_{2 n}, u\right) \\
& =\max \left\{1, d(S z, T u), 1, d^{1 / 2}(S z, T u), \min \{d(S z, T u), d(S z, T u), 1\}\right\} \\
& =d(S z, T u)
\end{aligned}
$$

Hence we have

$$
d(S z, T u) \leq d^{\lambda}(S z, T u)
$$

which implies that $T u=S z=z$. Since $B$ and $T$ are compatible of type $(B)$ and $B u=z=T u$, by Proposition 1.7, we have $T B u=B T u$ and so $B z=B T u=T B u=T z$.

Next putting $x=x_{2 n}$ and $y=z$ in $\left(C_{2}\right)$, we have

$$
d\left(S x_{2 n}, T z\right) \leq M^{\lambda}\left(x_{2 n}, z\right)
$$

where

$$
\begin{aligned}
& M\left(x_{2 n}, z\right) \\
& =\max \left\{d\left(A x_{2 n}, S x_{2 n}\right), d(B z, T z), d\left(B z, A x_{2 n}\right),\right. \\
& \quad\left(d\left(A x_{2 n}, T z\right) \cdot d\left(B z, S x_{2 n}\right)\right)^{1 / 2}, \min \left\{\frac{d\left(A x_{2 n}, S x_{2 n}\right) \cdot d(B z, T z)}{d\left(A x_{2 n}, B z\right)},\right. \\
& \left.\left.\quad \frac{d\left(A x_{2 n}, T z\right) \cdot d\left(B z, S x_{2 n}\right)}{d\left(A x_{2 n}, B z\right)}, \frac{d\left(A x_{2 n}, T z\right) \cdot d\left(B z, S x_{2 n}\right)}{d\left(S x_{2 n}, T z\right)}\right\}\right\} .
\end{aligned}
$$


Letting $n \rightarrow \infty$, we get

$$
\begin{aligned}
& \lim _{n \rightarrow \infty} M\left(x_{2 n}, z\right) \\
& =\max \{1,1, d(T z, z), d(z, T z), \min \{1 / d(z, T z), d(z, T z), d(T z, z))\}\} \\
& =d(z, T z) .
\end{aligned}
$$

Hence we have

$$
d(z, T z) \leq d^{\lambda}(z, T z)
$$

which implies $T z=z$. Since $T X \subset A X$, there exists a point $v \in X$ such that $z=T z=A v$.

Now putting $x=v$ and $y=z$ in $\left(C_{2}\right)$, we have

$$
d(S v, T z) \leq M^{\lambda}(v, z),
$$

where

$$
\begin{aligned}
& M(v, z) \\
& =\max \left\{d(A v, S v), d(B z, T z), d(B z, A v),(d(A v, T z) \cdot d(B z, S v))^{1 / 2},\right. \\
& \quad \min \left\{\frac{d(A v, S v) \cdot d(B z, T z)}{d(A v, B z)}, \frac{d(A v, T z) \cdot d(B z, S v)}{d(A v, B z)},\right. \\
& \left.\left.\quad \frac{d(A v, T z) \cdot d(B z, S v)}{d(S v, T z)}\right\}\right\} \\
& =\max \left\{d(T z, S v), 1,1, d^{1 / 2}(T z, S v), \min \{d(T z, S v), d(T z, S v), 1\}\right\} \\
& =d(T z, S v) .
\end{aligned}
$$

Hence we have

$$
d(S v, T z) \leq d^{\lambda}(T z, S v)
$$

which implies that $S v=T z=z$. Since $A$ and $S$ are compatible of type $(B)$ and $S v=z=A v$, it follows from Proposition 1.7 that $S z=S A v=A S v=A z$. Therefore, $A z=B z=S z=T z=z$ and hence $z$ is a common fixed point of $A, B, S$ and $T$.

Similarly, we can complete the proof when $T$ is continuous.

Now suppose that $A$ is continuous. Then we have

$$
A A x_{2 n} \rightarrow A z, \quad A S x_{2 n} \rightarrow A z \quad \text { as } n \rightarrow \infty .
$$

Since $A$ and $S$ are compatible of type $(B)$, it follows from Proposition 1.8 that

$$
S S x_{2 n} \rightarrow A z \quad \text { as } n \rightarrow \infty \text {. }
$$


Now putting $x=S x_{2 n}$ and $y=x_{2 n+1}$ in $\left(C_{2}\right)$, we have

$$
d\left(S S x_{2 n}, T x_{2 n+1}\right) \leq M^{\lambda}\left(S x_{2 n}, x_{2 n+1}\right),
$$

where

$$
\begin{aligned}
& M\left(S x_{2 n}, x_{2 n+1}\right) \\
& =\max \left\{d\left(A S x_{2 n}, S S x_{2 n}\right), d\left(B x_{2 n+1}, T x_{2 n+1}\right), d\left(B x_{2 n+1}, A S x_{2 n}\right),\right. \\
& \left(d\left(A S x_{2 n}, T x_{2 n+1}\right) \cdot d\left(B x_{2 n+1}, S S x_{2 n}\right)\right)^{1 / 2}, \\
& \min \left\{\frac{d\left(A S x_{2 n}, S S x_{2 n}\right) \cdot d\left(B x_{2 n+1}, T x_{2 n+1}\right)}{d\left(A S x_{2 n}, B x_{2 n+1}\right)},\right. \\
& \quad \frac{d\left(A S x_{2 n}, T x_{2 n+1}\right) \cdot d\left(B x_{2 n+1}, S S x_{2 n}\right)}{d\left(A S x_{2 n}, B x_{2 n+1}\right)}, \\
& \left.\left.\quad \frac{d\left(A S x_{2 n}, T x_{2 n+1}\right) \cdot d\left(B x_{2 n+1}, S S x_{2 n}\right)}{d\left(S S x_{2 n}, T x_{2 n+1}\right)}\right\}\right\} .
\end{aligned}
$$

Letting $n \rightarrow \infty$, we get

$$
\begin{aligned}
& \lim _{n \rightarrow \infty} M\left(S x_{2 n}, x_{2 n+1}\right) \\
& =\max \{1,1, d(z, A z), d(A z, z), \min \{1 / d(A z, z), d(A z, z), d(A z, z)\}\} \\
& =d(A z, z) .
\end{aligned}
$$

This implies that $A z=z$.

Now putting $x=z$ and $y=x_{2 n+1}$ in $\left(C_{2}\right)$, we have

$$
d\left(S z, T x_{2 n+1}\right) \leq M^{\lambda}\left(z, x_{2 n+1}\right),
$$

where

$$
\begin{aligned}
& M\left(z, x_{2 n+1}\right) \\
& =\max \left\{d(A z, S z), d\left(B x_{2 n+1}, T x_{2 n+1}\right), d\left(B x_{2 n+1}, A z\right),\right. \\
& \quad\left(d\left(A z, T x_{2 n+1}\right) \cdot d\left(B x_{2 n+1}, S z\right)\right)^{1 / 2}, \\
& \quad \min \left\{\frac{d(A z, S z) \cdot d\left(B x_{2 n+1}, T x_{2 n+1}\right)}{d\left(A z, B x_{2 n+1}\right)}, \frac{d\left(A z, T x_{2 n+1}\right) \cdot d\left(B x_{2 n+1}, S z\right)}{d\left(A z, B x_{2 n+1}\right)},\right. \\
& \left.\left.\quad \frac{d\left(A z, T x_{2 n+1}\right) \cdot d\left(B x_{2 n+1}, S z\right)}{d\left(S z, T x_{2 n+1}\right)}\right\}\right\} .
\end{aligned}
$$


Letting $n \rightarrow \infty$, we get

$$
\begin{aligned}
M(z, z)= & \max \left\{d(A z, S z), 1, d(z, A z), d^{1 / 2}(z, S z)\right. \\
& \quad \min \{d(z, S z), d(z, S z), 1\}\} \\
= & d(S z, z) .
\end{aligned}
$$

This implies that $S z=z$. Since $S X \subset B X$, there exists a point $w \in X$ such that $z=S z=B w$.

Next putting $x=z$ and $y=w$ in $\left(C_{2}\right)$, we have

$$
d(z, T w)=d(S z, T w) \leq M^{\lambda}(z, w)
$$

where

$$
\begin{aligned}
& M(z, w) \\
& =\max \left\{d(A z, S z), d(B w, T w), d(B w, A z),(d(A z, T w) \cdot d(B w, S z))^{1 / 2},\right. \\
& \quad \min \left\{\frac{d(A z, S z) \cdot d(B w, T w)}{d(A z, B w)}, \frac{d(A z, T w) \cdot d(B w, S z)}{d(A z, B w)},\right. \\
& \left.\left.\quad \frac{d(A z, T w) \cdot d(B w, S z)}{d(S z, T w)}\right\}\right\} \\
& =d(z, T w) .
\end{aligned}
$$

This implies that $z=T w$. Since $B$ and $T$ are compatible of type $(B)$ and $B w=$ $z=T w$, by Proposition 1.7, TBw=BTw and so $B z=B T w=T B w=T z$.

Now putting $x=z$ and $y=z$ in $\left(C_{2}\right)$, we have

$$
d(z, T z)=d(S z, T z) \leq M^{\lambda}(z, z)
$$

where

$$
\begin{aligned}
M(z, z)= & \max \{d(A z, S z), d(B z, T z), d(B z, A z), \\
& (d(A z, T z) \cdot d(B z, S z))^{1 / 2}, \min \left\{\frac{d(A z, S z) \cdot d(B z, T z)}{d(A z, B z)},\right. \\
& \left.\left.\frac{d(A z, T z) \cdot d(B z, S z)}{d(A z, B z)}, \frac{d(A z, T z) \cdot d(B z, S z)}{d(S z, T z)}\right\}\right\} \\
= & d(z, T z) .
\end{aligned}
$$

This implies that $z=T z$. Therefore, $z$ is a common fixed point of $A, B, S$ and $T$. 
Similarly, we can complete the proof when $B$ is continuous.

Finally, suppose that $z$ and $w(z \neq w)$ are two common fixed points.

Now putting $x=z$ and $y=w$ in $\left(C_{2}\right)$, we have

$$
d(z, w)=d(S z, T w) \leq M^{\lambda}(z, w)
$$

where

$$
\begin{aligned}
& M(z, w) \\
& =\max \left\{d(A z, S z), d(B w, T w), d(B w, A z),(d(A z, T w) \cdot d(B w, S z))^{1 / 2},\right. \\
& \quad \min \left\{\frac{d(A z, S z) \cdot d(B w, T w)}{d(A z, B w)}, \frac{d(A z, T w) \cdot d(B w, S z)}{d(A z, B w)},\right. \\
& \left.\left.\quad \frac{d(A z, T w) \cdot d(B w, S z)}{d(S z, T w)}\right\}\right\} \\
& =d(z, w),
\end{aligned}
$$

which implies that $z=w$. Therefore, $z$ is a unique common fixed point of $A, B, S$ and $T$. This completes the proof.

Next, we give the theorem for compatible mappings of type $(C)$.

Theorem 2.3. Let $A, B, S$ and $T$ be mappings of a complete multiplicative metric space $(X, d)$ into itself satisfying the conditions $\left(C_{1}\right),\left(C_{2}\right)$ and $\left(C_{3}\right)$.

Assume that the pairs $A, S$ and $B, T$ are compatible of type $(C)$. Then $A, B, S$ and $T$ have a unique common fixed point in $X$.

Proof. From the proof of Theorem 2.2, $\left\{y_{n}\right\}$ is a multiplicative Cauchy sequence in $X$ and hence it converges to some point $z \in X$. Further the subsequence $\left\{S x_{2 n}\right\},\left\{A x_{2 n}\right\},\left\{T x_{2 n+1}\right\}$ and $\left\{B x_{2 n+1}\right\}$ of $\left\{y_{n}\right\}$ converges to $z$.

Suppose that $S$ is continuous. Then

$$
S S x_{n} \rightarrow S z, \quad S A x_{2 n} \rightarrow S z \quad \text { as } n \rightarrow \infty
$$

Since $A$ and $S$ are compatible of type $(C)$, by Remark 1.9(2),

$$
A A x_{2 n} \rightarrow S u \quad \text { as } n \rightarrow \infty \text {. }
$$

Now putting $x=A x_{2 n}$ and $y=x_{2 n+1}$ in $\left(C_{2}\right)$, we have

$$
d\left(S A x_{2 n}, T x_{2 n+1}\right) \leq M^{\lambda}\left(A x_{2 n}, x_{2 n+1}\right),
$$


where

$$
\begin{aligned}
& M\left(A x_{2 n}, x_{2 n+1}\right) \\
& =\max \left\{d\left(A A x_{2 n}, S A x_{2 n}\right), d\left(B x_{2 n+1}, T x_{2 n+1}\right), d\left(B x_{2 n+1}, A A x_{2 n}\right),\right. \\
& \left(d\left(A A x_{2 n}, T x_{2 n+1}\right) \cdot d\left(B x_{2 n+1}, S A x_{2 n}\right)\right)^{1 / 2}, \\
& \quad \min \left\{\frac{d\left(A A x_{2 n}, S A x_{2 n}\right) \cdot d\left(B x_{2 n+1}, T x_{2 n+1}\right)}{d\left(A A x_{2 n}, B x_{2 n+1}\right)},\right. \\
& \quad \frac{d\left(A A x_{2 n}, T y\right) \cdot d\left(B x_{2 n+1}, S A x_{2 n}\right)}{d\left(A A x_{2 n}, B x_{2 n+1}\right)}, \\
& \left.\left.\quad \frac{d\left(A A x_{2 n}, T x_{2 n+1}\right) \cdot d\left(B x_{2 n+1}, S A x_{2 n}\right)}{d\left(S A x_{2 n}, T x_{2 n+1}\right)}\right\}\right\} .
\end{aligned}
$$

Taking $n \rightarrow \infty$, we get

$$
\begin{aligned}
& \lim _{n \rightarrow \infty} M\left(A x_{2 n}, x_{2 n+1}\right) \\
& =\max \{1,1, d(z, S z), d(S z, z), \min \{1 / d(S z, z), d(S z, z), d(S z, z)\}\} \\
& =d(S z, z),
\end{aligned}
$$

which implies that $S z=z$. Since $S X \subset B X$, there exists a point $u \in X$ such that $z=S z=B u$.

Consider $x=A x_{2 n}$ and $y=u$ in $\left(C_{2}\right)$, we have

$$
d\left(S A x_{2 n}, T u\right) \leq M^{\lambda}\left(A x_{2 n}, u\right),
$$

where

$$
\begin{aligned}
& M\left(A x_{2 n}, u\right) \\
& =\max \left\{d\left(A A x_{2 n}, S A x_{2 n}\right), d(B u, T u), d\left(B u, A A x_{2 n}\right),\right. \\
& \quad\left(d\left(A A x_{2 n}, T u\right) \cdot d\left(B u, S A x_{2 n}\right)\right)^{1 / 2}, \\
& \quad \min \left\{\frac{d\left(A A x_{2 n}, S A x_{2 n}\right) \cdot d(B u, T u)}{d\left(A A x_{2 n}, B u\right)}, \frac{d\left(A A x_{2 n}, T u\right) \cdot d\left(B u, S A x_{2 n}\right)}{d\left(A A x_{2 n}, B u\right)},\right. \\
& \left.\left.\quad \frac{d\left(A A x_{2 n}, T u\right) \cdot d\left(B u, S A x_{2 n}\right)}{d\left(S A x_{2 n}, T u\right)}\right\}\right\} .
\end{aligned}
$$

Taking $n \rightarrow \infty$, we get

$$
\begin{aligned}
& \lim _{n \rightarrow \infty} M\left(A x_{2 n}, u\right) \\
& =\max \left\{1, d(S z, T u), 1, d^{1 / 2}(T u, S z), \min \{d(T u, S z), d(T u, S z), 1\}\right\} \\
& =d(S z, T u) .
\end{aligned}
$$


Hence we have

$$
d(S z, T u) \leq d^{\lambda}(S z, T u)
$$

which implies that $T u=S z=z$. Since $B$ and $T$ are compatible of type $(C)$ and $B u=z=T u$, by Remark 1.9(1), we get $T B u=B T u$ and so $B z=B T u=$ $T B u=T z$.

Next putting $x=x_{2 n}$ and $y=z$ in $\left(C_{2}\right)$, we have

$$
d\left(S x_{2 n}, T z\right) \leq M^{\lambda}\left(x_{2 n}, z\right)
$$

where

$$
\begin{aligned}
& M\left(x_{2 n}, z\right) \\
& =\max \left\{d\left(A x_{2 n}, S x_{2 n}\right), d(B z, T z), d\left(B u, A x_{2 n}\right),\right. \\
& \quad\left(d\left(A x_{2 n}, T z\right) \cdot d\left(B z, S x_{2 n}\right)\right)^{1 / 2}, \min \left\{\frac{d\left(A x_{2 n}, S x_{2 n}\right) \cdot d(B z, T z)}{d\left(A x_{2 n}, B z\right)},\right. \\
& \left.\left.\quad \frac{d\left(A x_{2 n}, T z\right) \cdot d\left(B z, S x_{2 n}\right)}{d\left(A x_{2 n}, B z\right)}, \frac{d\left(A x_{2 n}, T z\right) \cdot d\left(B z, S x_{2 n}\right)}{d\left(S x_{2 n}, T z\right)}\right\}\right\} .
\end{aligned}
$$

Letting $n \rightarrow \infty$, we get

$$
\begin{aligned}
& \lim _{n \rightarrow \infty} M\left(x_{2 n}, z\right) \\
& =\max \left\{d(z, z), d(B z, T z), d(B z, z),(d(z, T z) \cdot d(B z, z))^{1 / 2}\right. \\
& \left.\quad \min \left\{\frac{d(z, z) \cdot d(B z, T z)}{d(z, B z)}, \frac{d(z, T z) \cdot d(B z, z)}{d(z, B z)}, \frac{d(z, T z) \cdot d(B z, z)}{d(z, T z)}\right\}\right\} \\
& =\max \{1,1, d(T z, z), d(T z, z), \min \{1 / d(z, T z), d(T z, z), d(T z, z)\}\} \\
& =d(T z, z) .
\end{aligned}
$$

This implies that $T z=z$. Since $T X \subset A X$, there exists a point $v \in X$ such that $z=T z=A v$.

Now putting $x=v$ and $y=z$ in $\left(C_{2}\right)$, we have

$$
d(S v, T z) \leq M^{\lambda}(v, z)
$$


where

$$
\begin{aligned}
& M(v, z) \\
& =\max \left\{d(A v, S v), d(B z, T z), d(B z, A v),(d(A v, T z) \cdot d(B z, S v))^{1 / 2},\right. \\
& \quad \min \left\{\frac{d(A v, S v) \cdot d(B z, T z)}{d(A v, B z)}, \frac{d(A v, T z) \cdot d(B z, S v)}{d(A v, B z)},\right. \\
& \left.\left.\quad \frac{d(A v, T z) \cdot d(B z, S v)}{d(S v, T z)}\right\}\right\} \\
& =\max \left\{d(T z, S v), 1,1, d^{1 / 2}(T z, S v), \min \{d(T z, S v), 1,1\}\right\} \\
& =d(T z, S v) .
\end{aligned}
$$

Hence we have

$$
d(S v, T z) \leq d^{\lambda}(T z, S v)
$$

which implies that $S v=T z=z$. Since $A$ and $S$ are compatible of type $(C)$ and $S v=z=A v$, it follows from Remark 1.9(1) that $S z=S A v=A S v=A z$. Therefore $B z=A z=T z=S z=z$ and hence $z$ is a common fixed point of $A, B, S$ and $T$.

Similarly, we can complete the proof when $A$ or $B$ or $T$ is continuous.

Uniqueness follows easily. Therefore $A, B, S$ and $T$ have a unique common fixed point in $X$. This completes the proof.

Finally, we give the theorem for compatible mappings of type $(P)$.

Theorem 2.4. Let $A, B, S$ and $T$ be mappings of a complete multiplicative metric space $(X, d)$ into itself satisfying the conditions $\left(C_{1}\right),\left(C_{2}\right)$ and $\left(C_{3}\right)$.

Assume that the pairs $A, S$ and $B, T$ are compatible of type $(P)$. Then $A, B, S$ and $T$ have a unique common fixed point in $X$.

Proof. From the proof of Theorem 2.2, $\left\{y_{n}\right\}$ is a multiplicative Cauchy sequence in $X$ and hence it converges to some point $z \in X$. Further the subsequence $\left\{S x_{2 n}\right\},\left\{A x_{2 n}\right\},\left\{T x_{2 n+1}\right\}$ and $\left\{B x_{2 n+1}\right\}$ of $\left\{y_{n}\right\}$ converges to $z$.

Suppose that $S$ is continuous. Then

$$
S S x_{2 n} \rightarrow S z, \quad S A x_{2 n} \rightarrow S z \quad \text { as } n \rightarrow \infty
$$

Since $A$ and $S$ are compatible of type $(P)$, it follows from Remark 1.9(2) that

$$
S S x_{2 n} \rightarrow A z \quad \text { as } n \rightarrow \infty
$$


Now putting $x=A x_{2 n}$ and $y=x_{2 n+1}$ in $\left(C_{2}\right)$, we have

$$
d\left(S A x_{2 n}, T x_{2 n+1}\right) \leq M^{\lambda}\left(A x_{2 n}, x_{2 n+1}\right),
$$

where

$$
\begin{aligned}
M( & \left.A x_{2 n}, x_{2 n+1}\right) \\
= & \max \left\{d\left(A A x_{2 n}, S A x_{2 n}\right), d\left(B x_{2 n+1}, T x_{2 n+1}\right), d\left(B x_{2 n+1}, A A x_{2 n}\right),\right. \\
& \left(d\left(A A x_{2 n}, T x_{2 n+1}\right) \cdot d\left(B x_{2 n+1}, S A x_{2 n}\right)\right)^{1 / 2}, \\
& \min \left\{\frac{d\left(A A x_{2 n}, S A x_{2 n}\right) \cdot d\left(B x_{2 n+1}, T x_{2 n+1}\right)}{d\left(A A x_{2 n}, B x_{2 n+1}\right)},\right. \\
& \frac{\left(d\left(A A x_{2 n}, T x_{2 n+1}\right) \cdot d\left(B x_{2 n+1}, S A x_{2 n}\right)\right.}{d\left(A A x_{2 n}, B x_{2 n+1}\right)}, \\
& \left.\left.\frac{d\left(A A x_{2 n}, T x_{2 n+1}\right) \cdot d\left(B x_{2 n+1}, S A x_{2 n}\right)}{d\left(S A x_{2 n}, T x_{2 n+1}\right)}\right\}\right\} .
\end{aligned}
$$

Taking $n \rightarrow \infty$, we get

$$
\begin{aligned}
& \lim _{n \rightarrow \infty} M\left(A x_{2 n}, x_{2 n+1}\right) \\
& =\max \left\{d(S z, S z), d(z, z), d(z, S z),(d(S z, z) \cdot d(z, S z))^{1 / 2},\right. \\
& \quad \min \{1 / d(S z, z), d(S z, z), d(S z, z)\}\} \\
& =d(S z, z) .
\end{aligned}
$$

Hence we have

$$
d(S z, z) \leq d^{\lambda}(S z, z)
$$

which implies that $S z=z$. Since $S X \subset B X$, there exists a point $u \in X$ such that $z=S z=B u$.

Consider $x=A x_{2 n}$ and $y=u$ in $\left(C_{2}\right)$, we have

$$
d\left(S A x_{2 n}, T u\right) \leq M^{\lambda}\left(A x_{2 n}, u\right)
$$

where

$$
\begin{aligned}
& M\left(A x_{2 n}, u\right) \\
& =\max \left\{d\left(A A x_{2 n}, S A x_{2 n}\right), d(B u, T u), d\left(B u, A A x_{2 n}\right),\right. \\
& \quad\left(d\left(A A x_{2 n}, T u\right) \cdot d\left(B u, S A x_{2 n}\right)\right)^{1 / 2}, \\
& \quad \min \left\{\frac{d\left(A A x_{2 n}, S A x_{2 n}\right) \cdot d(B u, T u)}{d\left(A A x_{2 n}, B u\right)}, \frac{d\left(A A x_{2 n}, T u\right) \cdot d\left(B u, S A x_{2 n}\right)}{d\left(A A x_{2 n}, B u\right)},\right. \\
& \left.\left.\quad \frac{d\left(A A x_{2 n}, T u\right) \cdot d\left(B u, S A x_{2 n}\right)}{d\left(S A x_{2 n}, T u\right)}\right\}\right\} .
\end{aligned}
$$


Taking $n \rightarrow \infty$, we get

$$
\begin{aligned}
& \lim _{n \rightarrow \infty} M\left(A x_{2 n}, u\right) \\
& =\max \left\{d(S z, S z), d(S z, T u), d(T u, S z),(d(S z, T u) \cdot d(B u, S z))^{1 / 2},\right. \\
& \quad \min \left\{\frac{d(S z, S z) \cdot d(S z, T u)}{d(S z, B u)}, \frac{d(S z, T u) \cdot d(T u, S z)}{d(S z, B u)},\right. \\
& \left.\left.\quad \frac{d(S z, T u) \cdot d(S z, T u)}{d(S z, T u)}\right\}\right\} \\
& =d(S z, T u) .
\end{aligned}
$$

Hence we have

$$
d(S z, T u) \leq d^{\lambda}(S z, T u)
$$

which implies that $T u=S z=z=B u$. Since $B$ and $T$ are compatible of type $(P)$, by Remark 1.9(1), we have $B z=B B u=T T u=T z$.

Now putting $x=x_{2 n}$ and $y=z$ in $\left(C_{2}\right)$, we have

$$
d\left(S x_{2 n}, T z\right) \leq M^{\lambda}\left(x_{2 n}, z\right),
$$

where

$$
\begin{aligned}
& M\left(x_{2 n}, z\right) \\
& =\max \left\{d\left(A x_{2 n}, S x_{2 n}\right), d(B z, T z), d\left(B z, A x_{2 n}\right),\right. \\
& \quad\left(d\left(A x_{2 n}, T z\right) \cdot d\left(B z, S x_{2 n}\right)\right)^{1 / 2}, \min \left\{\frac{d\left(A x_{2 n}, S x_{2 n}\right) \cdot d(B z, T z)}{d\left(A x_{2 n}, B z\right)},\right. \\
& \left.\left.\quad \frac{d\left(A x_{2 n}, T z\right) \cdot d\left(B z, S x_{2 n}\right)}{d\left(A x_{2 n}, B z\right)}, \frac{d\left(A x_{2 n}, T z\right) \cdot d\left(B z, S x_{2 n}\right)}{d\left(S x_{2 n}, T z\right)}\right\}\right\} .
\end{aligned}
$$

Letting $n \rightarrow \infty$, we get

$$
\begin{aligned}
& \lim _{n \rightarrow \infty} M\left(x_{2 n}, z\right) \\
& =\max \left\{d(z, z), d(B z, T z), d(B z, z),(d(z, T z) \cdot d(B z, z))^{1 / 2}\right. \\
& \left.\quad \min \left\{\frac{d(z, z) \cdot d(B z, T z)}{d(z, B z)}, \frac{d(z, T z) \cdot d(B z, z)}{d(z, B z)}, \frac{d(z, T z) \cdot d(B z, z)}{d(z, T z)}\right\}\right\} \\
& =\max \{1,1, d(T z, z), d(T z, z), \min \{1 / d(z, T z), d(T z, z), d(T z, z)\}\} \\
& =d(T z, z) .
\end{aligned}
$$


Hence we have

$$
d(z, T z) \leq d^{\lambda}(T z, z)
$$

which implies that $B z=T z=z$. Since $T X \subset A X$, so there exists a point $v \in X$ such that $z=T z=A v$.

On putting $x=v$ and $y=z$ in $\left(C_{2}\right)$, we have

$$
d(S v, T z) \leq M^{\lambda}(v, z)
$$

where

$$
\begin{aligned}
M(v, z)= & \max \{d(A v, S v), d(B z, T z), d(B z, A v) \\
& (d(A v, T z) \cdot d(B z, S v))^{1 / 2}, \min \left\{\frac{d(A v, S v) \cdot d(B z, T z)}{d(A v, B z)},\right. \\
& \left.\left.\frac{d(A v, T z) \cdot d(B z, S v)}{d(A v, B z)}, \frac{d(A v, T z) \cdot d(B z, S v)}{d(S v, T z)}\right\}\right\} \\
= & \max \left\{d(T z, S v), 1,1, d^{1 / 2}(T z, S v), \min \{d(T z, S v), 1,1\}\right\} \\
= & d(T z, S v) .
\end{aligned}
$$

Hence we have

$$
d(S v, T z) \leq d^{\lambda}(T z, S v)
$$

which implies that $S v=T z=z=A v$. Since $A$ and $S$ are compatible of type $(P)$, by Remark 1.9(1), we have $S z=S S v=A A v=A z$. Since $A z=B z=$ $S z=T z=z$, we get $z$ is a common fixed point of $A, B, S$ and $T$.

Similarly, we can complete the proof when $A$ or $B$ or $T$ is continuous.

Uniqueness follows easily. Therefore $A, B, S$ and $T$ have a unique common fixed point in $X$. This completes the proof.

\section{Acknowledgments}

This work was supported by the Dong-A University research fund.

\section{References}

[1] A.E. Bashirov, E.M. Kurplnara, A. Ozyapici, Multiplicative calculus and its applicatiopns, J. Math. Anal. Appl., 337 (2008), 36-48, doi: 10.1016/j.jmaa.2007.03.081.

[2] M. Özavsar, A.C. Çevikel, Fixed points of multiplicative contraction mappings on multiplicative metric spaces, ArXiv:1205.5131v1 [math.GM] (2012), 14 pages. 
[3] S.M. Kang, P. Kumar, S. Kumar, P. Nagpal, S.K. Garg, Common fixed points for compatible mappings and its variants in multiplicative metric spaces, Int. J. Pure Appl. Math., 102 (2015), 383-406. doi: 10.12732/ijpam.v102i2.14

[4] P. Kumar, S. Kumar, S.M. Kang, C.Y. Jung, Fixed points for compatible mappings in multiplicative metric spaces, Appl. Math. Sci., Submitted. 